\title{
The ERG in drug development: translation from animal models to human
}

\author{
Mitchell Brigell
}

Received: 7 December 2008/Accepted: 15 December 2008/Published online: 8 January 2009

(C) Springer-Verlag 2008

Drug development is a long and complex process with a low likelihood of success. In order to allocate resources to compounds that will provide significant improvement to patients, it is imperative to develop measures that are predictive of clinical efficacy and safety. In the development of therapies for diseases of the retina, the electroretinogram (ERG) is a technique that is well suited to provide such information. As a functional measure, the ERG is more closely related to accepted behavioral endpoints than structural measures such as optical coherence tomography, and fluorescein angiography. Unlike most other measures of visual function, the ERG is easily obtained in animal models. These characteristics make the ERG a good biomarker to suggest the safety and efficacy of new treatments of retinal diseases. The ERG is also a sensitive measure of toxic effects of systemic therapies on the retina.

In 2007, I organized a symposium entitled $E R G$ in Drug Development: Translation from Animal Models to Human, held in Hyderabad, India as a part of the 45th ISCEV Symposium. The papers that follow summarize some of the presentations made at this symposium. Ido Perlman gave the keynote address at the Drug Development symposium, summarizing his elegant work using the ERG to detect and understand toxic effects of compounds on the retina. In this paper, Dr. Perlman also shows how these studies can help us understand the normal functioning of the retina. Jonathan Lyons and Matt Severns extend their published work on the use of the mfERG and ring ratios to detect toxic effects of hydroxychloroquine on the retinae of patients on long-term high-dose therapy. Pierre LaChapelle et al. show the use of the ERG in animal models of retinopathy of prematurity, and Ann Fulton et al. summarize their use of the ERG in translational research on degenerative and ischemic retinal diseases in children. Finally, Christina Gerth provides an overview of the use of the ERG in agerelated macular degeneration and the potential role of the mfERG in monitoring therapeutic interventions in this disease. Other presentations at the symposium were by Mathias Seeliger on the use of the ERG in animal models of retinal degenerative diseases, Laura Frishman on the use of various ERG techniques in preclinical models of glaucoma, Michael Bach on use of the pattern ERG in longitudinal studies of patients with ocular hypertension, and Eberhardt Zrenner emphasizing the need for standardized ERG protocols for use in multi-center clinical trials. Abstracts of all presentations in this special symposium can be found in Documenta Ophthalmologica 2007;115:21-26.

M. Brigell ( $\bowtie)$

Novartis Institutes for Biomedical Research, Cambridge, MA, USA

e-mail: Mitchell.Brigell@Novartis.com 